\title{
Twist of fractional oscillations
}

\author{
Aleksander A. Stanislavsky* \\ Institute of Radio Astronomy, 4 Chervonopraporna St., Kharkov 61002, Ukraine
}

\begin{abstract}
Using the method of the Laplace transform, we consider fractional oscillations. They are obtained by the time-clock randomization of ordinary harmonic vibrations. In contrast to sine and cosine, the functions describing the fractional oscillations exhibit a finite number of damped oscillations with an algebraic decay. Their fractional differential equation is derived.
\end{abstract}

Key words:

Oscillation, Laplace transform, Decomposition, Fractional differential equation, Mittag-Leffler function

\section{Introduction}

According to fractional calculus the harmonic oscillator is a particular case of the fractional oscillator [1,2,3. It suffices to say that the fractional oscillator equation is a generalization of the classical harmonic equation by replacing the second-order derivative by a fractional one. If the harmonic oscillator is described by elementary harmonic functions, the fractional oscillator solution is expressed in terms of Mittag-Leffler functions. The interrelation between these oscillators has clarified with the consideration of temporal subordination. Then the development of an oscillatory process is governed by its own internal clock (operational time) that is not synchronized with physical (deterministic) clock [4]. As has been shown in [5] that the fractional oscillator results from an ensemble average of harmonic oscillations under stochastic time arrow. In fact, the fractional oscillator can be considered as a time-clock randomization (subordination) of the conventional harmonic oscillator. The new time clock is the continuous limit of the discrete counting process, when

\footnotetext{
* E-mail: alexstan@ri.kharkov.ua
} 
the time variable is a sum of random temporal intervals belonging to an $\alpha$ stable distribution. Hence there exists a direct correspondence between the functions of both oscillators.

In particular, the cosine function $\cos (t)$ is connected with the one-parameter Mittag-Leffler function $E_{\alpha}\left(-t^{\alpha}\right)$. Its features has been studied in detail (see, for example, [1] and references therein). Passing to the limit $\alpha \rightarrow 2$, this Mittag-Leffler function transforms to $\cos (t)$. One of very interesting properties of the one-parameter Mittag-Leffler function is a finite number of zeros. Therefore, the function has a finite number of damped oscillations with an algebraic decay. For completeness it is necessary to investigate also the "sine" correspondence of fractional oscillations. The function is briefly mentioned in [3] without any detailed analysis. In this connection it should be pointed out that the displacement of harmonic oscillator and its momentum are written by means of ordinary harmonic functions. Similar values (displacement and momentum) may be formulated for fractional oscillator too. Their consideration is of great interest. They are just expressed in terms of Mittag-Leffler functions mentioned above. The aim of this paper is to study their features in the context of fractional oscillator.

The paper is organized as follows. In Section 2 we start our analysis with the time-clock randomization of the ordinary harmonic oscillator. This allows us to use the integral relation between the harmonic functions and the Mittag-Leffler functions of the fractional oscillator. Our main interest will be focused on the Mittag-Leffler function having the sine function as a limit. Next we investigate its properties in Section 3 and derive a fractional differential equation for which this function is its solution (Section 4). As a comparison, the "cosine" correspondence of fractional oscillations will be given simultaneously. Section 5 is devoted to a consideration of fractional oscillation zeros.

\section{Probabilistic point of view}

If one randomizes the time clock in accordance with [5], the fractional oscillations are written by means of

$$
\begin{aligned}
& A(t)=\int_{0}^{\infty} p^{S}(t, \tau) \cos \omega \tau d \tau=E_{\alpha, 1}\left(-\omega^{2} t^{\alpha}\right)=E_{\alpha}\left(-\omega^{2} t^{\alpha}\right), \\
& B(t)=\int_{0}^{\infty} p^{S}(t, \tau) \sin \omega \tau d \tau=\omega t^{\alpha / 2} E_{\alpha, 1+\alpha / 2}\left(-\omega^{2} t^{\alpha}\right),
\end{aligned}
$$


where $0<\alpha<2$,

$$
E_{\mu, \nu}(z)=\sum_{k=0}^{\infty} \frac{z^{k}}{\Gamma(\mu k+\nu)}, \quad \mu, \nu>0
$$

is the Mittag-Leffler function, $\Gamma(z)$ the gamma function, and

$$
p^{S}(t, \tau)=\frac{1}{2 \pi j} \int_{B r} e^{s t-\tau s^{\alpha / 2}} s^{-1+\alpha / 2} d s
$$

determines the probability to be at the internal time $\tau$ on the real time $t$. Here $B r$ denotes the Bromwich path (a line $\operatorname{Re} s=\sigma$ with a value $\sigma \geq 1$ ), and $j=\sqrt{-1}$.

Since the function $A(t)$ has been reviewed in [1], we devote our attention more to the function $B(t)$. The latter has a Laplace inversion integral

$$
B(t)=\frac{\omega}{2 \pi j} \int_{B r} e^{s t} \frac{s^{-1+\alpha / 2}}{s^{\alpha}+\omega^{2}} d s .
$$

The fractional oscillations exist for $1<\alpha<2$. It is that the case is of interest to us and will be discussed below.

For convenience one puts $\omega=1$, then one denotes $B(t)$ as $i_{\alpha}(t)$. It should be pointed out that for $\omega=1$ the function $A(t)$ is equal to $e_{\alpha}(t)$ according to the notations of [1]. As the Laplace image of the function $e_{\alpha}(t)=$ $\sum_{k=0}^{\infty}\left(-t^{\alpha}\right)^{k} / \Gamma(\alpha k+1)$ is $s^{\alpha-1} /\left(1+s^{\alpha}\right)$, it is easy to determine the relation between $e_{\alpha}(t)$ and $i_{\alpha}(t)$ via the fractional integral of $\alpha / 2$ order, namely

$$
i_{\alpha}(t)=J^{\alpha / 2} e_{\alpha}(t)=\frac{1}{\Gamma(\alpha / 2)} \int_{0}^{t}(t-\tau)^{-1+\alpha / 2} e_{\alpha}(\tau) d \tau
$$

Recall that for $\alpha=1,2$ the function $e_{\alpha}(t)$ takes the form

$$
e_{1}(t)=e^{-t}, \quad e_{2}(t)=\cos t
$$

Define the same in reference to the function $i_{\alpha}(t)$ for $\alpha=1,2$. At once it is obvious that $i_{2}(t)=\sin t$. Using the Efros' theorem, we find $i_{1}(t)$ via the Laplace image

$$
i_{1}(t)=\frac{1}{2 \pi j} \int_{B r} e^{s t} \frac{d s}{\sqrt{s}(s+1)}=\frac{1}{\sqrt{\pi t}} \int_{0}^{\infty} e^{-\tau^{2} /(4 t)} \sin \tau d \tau=e^{-t} \operatorname{erfi}(\sqrt{t}) .
$$




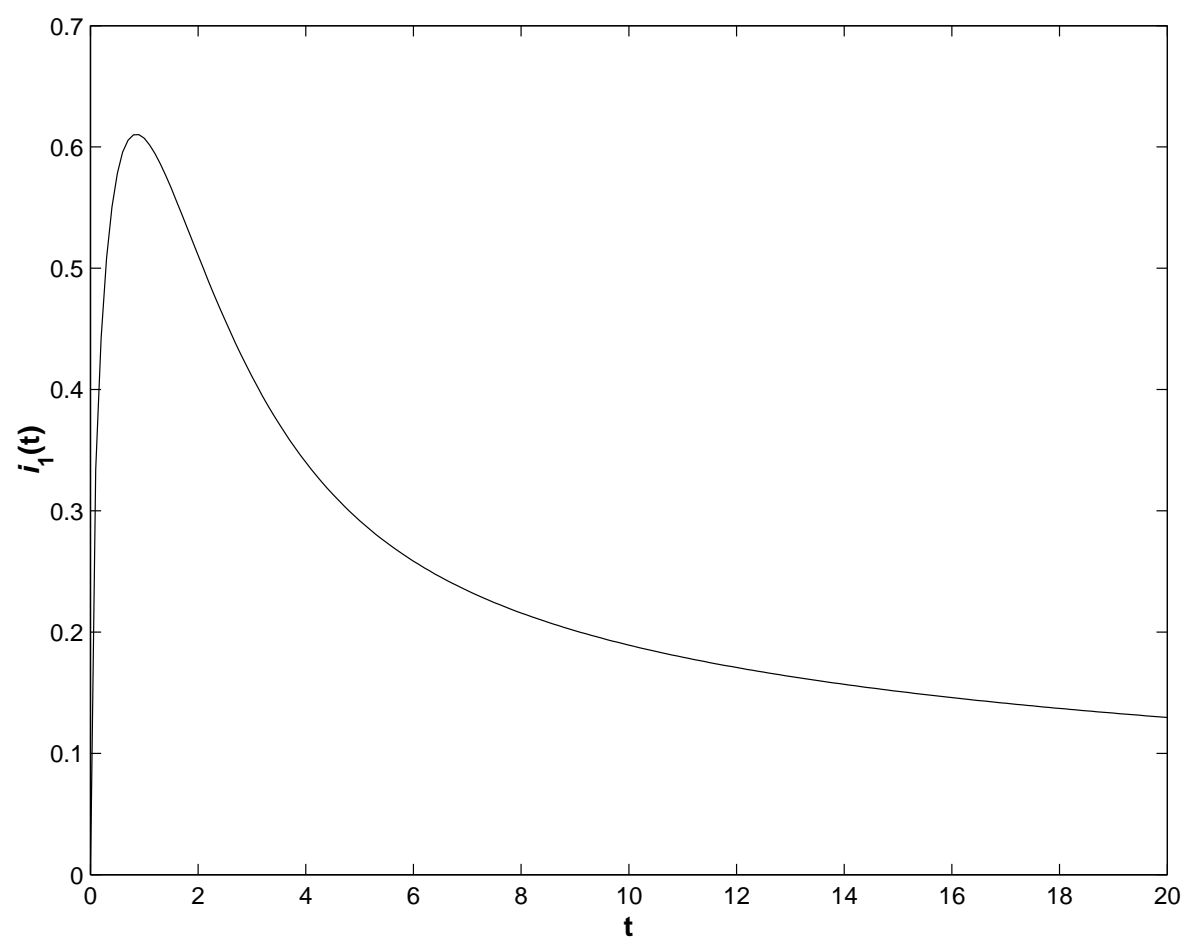

Fig. 1. Fractional one-half-order integral of the exponential function, $i_{1}(t)=J^{1 / 2} \exp (t)$.

Note that $\operatorname{erfi}(x)=\frac{2}{\sqrt{\pi}} \int_{0}^{x} \exp \left(y^{2}\right) d y$ is the imaginary error function [6]. It is a real-valued, entire function defined by $\operatorname{erfi}(x)=\operatorname{erf}(j x) / j$. In contrast to the ordinary error function $\operatorname{erf}(x)=\frac{2}{\sqrt{\pi}} \int_{0}^{x} \exp \left(-y^{2}\right) d y$, the imaginary error function is not bounded, but the function $i_{1}(t)$ is bounded. It tends to zero in the limit $t \rightarrow \infty$. Moreover, the latter is very close to the Dawson's integral $D(x)=e^{-x^{2}} \int_{0}^{x} \exp \left(y^{2}\right) d y$ [7]. In this connection it should be mentioned the asymptotic behavior of the imaginary error function

$$
\operatorname{erfi}(x)=\frac{1}{\sqrt{\pi}} e^{x^{2}}\left(x^{-1}+\frac{1}{2} x^{-3}+\frac{3}{4} x^{-5}+\frac{15}{8} x^{-7}+\ldots\right) .
$$

It may be advantageous for the estimation of $i_{1}(t)$ with $t \gg 1$. To sum up above is the numerical simulation of $i_{1}(t)$ represented in Fig. 1 ,

\section{Decomposition}

The next important step consists in the decomposition $i_{\alpha}(t)$ into two contributions. For this purpose we bow the Bromwich path of integration into the equivalent Hankel path. Then the loop will start from minus infinity along the lower side of negative real axis, encircle $|s|=1$ counter-clockwise and end at 


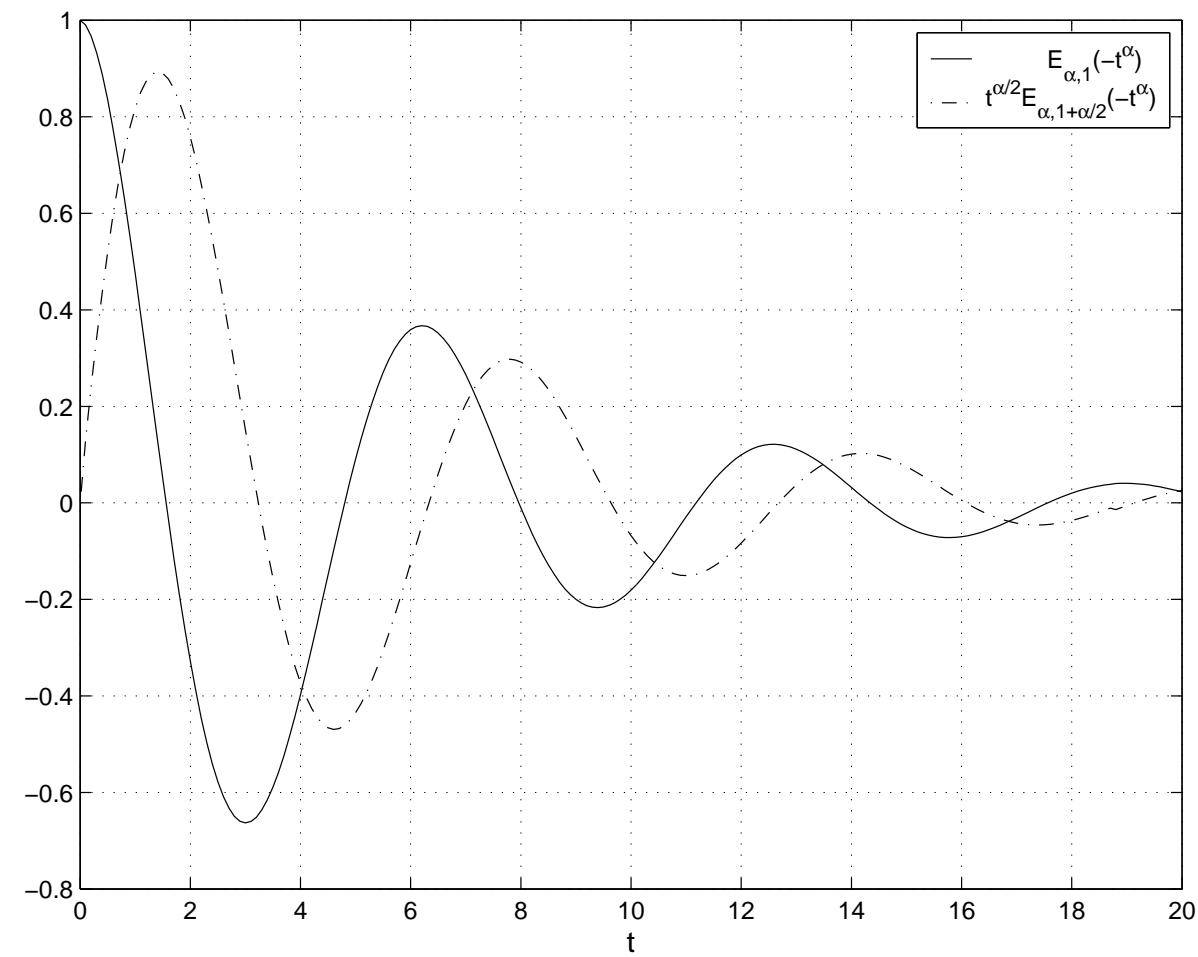

Fig. 2. Fractional oscillations for $\alpha=1.8$.

minus infinity along the upper side of the negative real axis. The first contribution arises from two borders of the cut negative real axis. Taking $s=r e^{j \pi}$ along the upper border and $s=r e^{-j \pi}$ along the lower border, we get

$$
h_{\alpha}(t)=\int_{0}^{\infty} e^{-r t} V_{\alpha}(r) d r
$$

with

$$
V_{\alpha}(r)=\frac{1}{\pi} \frac{r^{-1+\alpha / 2}\left(1-r^{\alpha}\right) \sin (\pi \alpha / 2)}{r^{2 \alpha}+2 r^{\alpha} \cos (\pi \alpha)+1} .
$$

The part $h_{\alpha}(t)$ is not completely monotonic, as the function $V_{\alpha}(r)$ takes on both positive and negative values (see Theorem 7 in [8]). It becomes vanishingly small with $t$ tending to infinity. The second contribution is calculated by means of residues. The poles $s_{0}=\exp (j \pi / \alpha)$ and $s_{1}=\exp (-j \pi / \alpha)$ give

$$
q_{\alpha}(t)=\frac{2}{\alpha} e^{t \cos (\pi / \alpha)} \sin \left[t \sin \left(\frac{\pi}{\alpha}\right)\right] .
$$

This part clearly demonstrates an oscillatory character. Due to it, the oscillatory behavior is carried over to the function $i_{\alpha}(t)$ itself. 
It is useful to remark that the functions $i_{\alpha}(t)$ resembles $e_{\alpha}(t)$. So for $1<\alpha<2$ the function $e_{\alpha}(t)$ also consists of two parts, $e_{\alpha}(t)=f_{\alpha}(t)+g_{\alpha}(t)$. From [1] it follows that the monotonic part is

$$
f_{\alpha}(t)=\int_{0}^{\infty} e^{-r t} K_{\alpha}(r) d r
$$

where

$$
K_{\alpha}(r)=\frac{1}{\pi} \frac{r^{\alpha-1} \sin (\pi \alpha)}{r^{2 \alpha}+2 r^{\alpha} \cos (\pi \alpha)+1}
$$

According to [8], the function $f_{\alpha}(t)$ is complete monotonic. The second contribution of $e_{\alpha}(t)$ takes the oscillatory form

$$
g_{\alpha}(t)=\frac{2}{\alpha} e^{t \cos (\pi / \alpha)} \cos \left[t \sin \left(\frac{\pi}{\alpha}\right)\right]
$$

To have a clear idea of $i_{\alpha}(t)$ and $e_{\alpha}(t)$, their pictures are represented in Fig. 2 .

\section{Equations of fractional oscillations}

The first approach to fractional oscillations had enough a formal character. It proceeded from the simple change of the second derivative in the harmonic oscillator equation to the derivative of a fractional one. In other words, such a equation was postulated. After solving it the fractional oscillation features were established. Nevertheless, there is an alternative way to fractional calculus [9]. The probabilistic point of view shows that the derivative of fractional order is connected with $\alpha$-stable probability distributions [10]. Starting from the analysis of stochastic random processes, this permits ones to derive the fractional differential equations responsible for fractional oscillations. Here we intend to give new examples demonstrating this approach.

Let the sign $\div$ be for the juxtaposition of a function depending on $t$ with its Laplace image depending on $s$, namely

$$
u(t) \quad \div \quad \bar{u}(s)=\int_{0}^{\infty} e^{-s t} u(t) d t
$$


The reader is reminded that

$$
e_{\alpha}(t) \quad \div \quad \frac{s^{\alpha-1}}{s^{\alpha}+1}
$$

If the Laplace image is written as

$$
\frac{s^{\alpha-1}}{s^{\alpha}+1}=\frac{1}{s} \frac{1}{\left(1+1 / s^{\alpha}\right)},
$$

the expression

$$
\bar{e}_{\alpha}(s)+\frac{1}{s^{\alpha}} \bar{e}_{\alpha}(s)=\frac{1}{s}
$$

is treated by a (fractional) integral equation after the Laplace transform. Since $e_{\alpha}(0)=1$, from the Laplace inversion we find

$$
e_{\alpha}(t)=1-J^{\alpha} e_{\alpha}(t)
$$

According to [11, the definition of fractional derivative is

$$
\tilde{D}^{\alpha} u(t):=J^{m-\alpha} D^{m} u(t)
$$

taking the positive integer number $m$ with $m-1<\alpha \leq m$. In fact, $D^{m}$ is the $m$-derivative under the integral $J^{m-\alpha}$. Then the function $e_{\alpha}(t)$ satisfies also the equivalent equation in the differential form

$$
\tilde{D}^{\alpha} e_{\alpha}(t)+e_{\alpha}(t)=0
$$

It should be pointed out, though this equation was known earlier, it was not derived but postulated. Using the approach, now we intend to derive an equation describing $i_{\alpha}(t)$.

In order to obtain the equation for $i_{\alpha}(t)$, we need to consider its Laplace image

$$
i_{\alpha}(t) \quad \div \quad \frac{1}{s} \frac{1}{\left(s^{\alpha / 2}+s^{-\alpha / 2}\right)}
$$

The initial condition is $i_{\alpha}(0)=0$. Thus, the corresponding equation becomes

$$
\tilde{D}^{\alpha / 2} i_{\alpha}(t)+J^{\alpha / 2} i_{\alpha}(t)=1
$$


It is interesting to observe that following the argument of [3], the generalized momentum of the fractional oscillator takes the form

$$
p_{\alpha}=m \tilde{D}^{\alpha / 2} q_{\alpha}(t)=-m q_{\alpha}(0) \omega^{2} t^{\alpha / 2} E_{\alpha, 1+\alpha / 2}\left(-\omega^{2} t^{\alpha}\right)
$$

where $q_{\alpha}$ is the displacement, $\omega$ the circular frequency, $m$ the generalized mass. To put it in another way, the momentum is expressed in terms of $-i_{\alpha}(t)$. Really, expanding $e_{\alpha}(t)$ and $i_{\alpha}(t)$ in an infinite power series about $t^{\alpha}$ and differentiating the functions by $\tilde{D}^{\alpha / 2}$ with respect to $t$, we obtain

$$
\tilde{D}^{\alpha / 2} e_{\alpha}(t)=-i_{\alpha}(t), \quad \tilde{D}^{\alpha / 2} i_{\alpha}(t)=e_{\alpha}(t)
$$

whereas

$$
J^{\alpha / 2} e_{\alpha}(t)=i_{\alpha}(t), \quad J^{\alpha / 2} i_{\alpha}(t)=1-e_{\alpha}(t) .
$$

Consequently, Eq.(41) is reduced to

$$
\tilde{D}^{\alpha} i_{\alpha}(t)+i_{\alpha}(t)=0 \text {. }
$$

The form of this equation coincides with Eq.(3). Thus, the functions $e_{\alpha}(t)$ and $i_{\alpha}(t)$ represent two independent solutions of the same equation.

Moreover, this permits one to derive the equation of fractional oscillator by means of the Hamilton formalism with some alterations. So the generalized Hamiltonian is written as

$$
\mathcal{H}_{\alpha}=\left(p_{\alpha}^{2}+\omega^{2} q_{\alpha}^{2}\right) / 2
$$

It corresponds to the total energy of the fractional oscillator [3]. In the Hamilton form the motion equation of fractional oscillator becomes

$$
\begin{aligned}
\tilde{D}^{\alpha / 2} q_{\alpha} & =\frac{d^{\alpha / 2} q_{\alpha}}{d t^{\alpha / 2}}=\frac{\partial \mathcal{H}_{\alpha}}{\partial p_{\alpha}}=p_{\alpha} \\
\tilde{D}^{\alpha / 2} p_{\alpha} & =\frac{d^{\alpha / 2} p_{\alpha}}{d t^{\alpha / 2}}=-\frac{\partial \mathcal{H}_{\alpha}}{\partial q_{\alpha}}=-\omega^{2} q_{\alpha} .
\end{aligned}
$$

The modification of the conventional representation of the Hamilton equations is conditioned on a random interaction of the system of harmonic oscillators with environment. Each harmonic oscillator is governed by its own internal clock. Although its dynamics is described by the ordinary Hamiltonian equations, $p(\tau)$ and $q(\tau)$ depend on the operational time. The passage from the 
operational time to the physical time via the averaging procedure accounts for the interaction of the system of harmonic oscillators with environment. This results in this system on the whole behaving as a fractional oscillator. The momentum of the fractional oscillator and its displacement take the integral form

$$
p_{\alpha}(t)=\int_{0}^{\infty} p^{S}(t, \tau) p(\tau) d \tau, \quad q_{\alpha}(t)=\int_{0}^{\infty} p^{S}(t, \tau) q(\tau) d \tau .
$$

Therefore, the fractional oscillator is an ensemble average of harmonic oscillations (see details in [5]). Although the Hamiltonian (5) is not an explicit function of time, for non-integer values $\alpha$ the dynamic system is nonconservative because of the fractional derivative of momentum.

As is well known [12, the Laplace images are also useful for finding an asymptotic behavior of transformed functions. With this in mind we expand the term $1 /\left(s^{\alpha}+1\right)$ in a series with respect to $s$. This technique works finely for the solutions of linear fractional differential equations [1]13]. Taking the leading terms, we obtain the asymptotic representations as $t \rightarrow \infty$, namely

$$
e_{\alpha}(t) \sim \frac{t^{-\alpha}}{\Gamma(1-\alpha)}, \quad i_{\alpha}(t) \sim \frac{t^{-\alpha / 2}}{\Gamma(1-\alpha / 2)}
$$

For $1<\alpha<2$ the denominator $\Gamma(1-\alpha)$ is less than zero, whereas $\Gamma(1-$ $\alpha / 2)>0$. It is easy to show that the asymptotic algebraic decay of $e_{\alpha}(t)$ and $i_{\alpha}(t)$ is completely determined by the parts $f_{\alpha}(t)$ and $h_{\alpha}(t)$, respectively (see Section (3). In the classical case $\alpha=2$ the contributions $f_{\alpha}(t)$ and $h_{\alpha}(t)$ are equal to zero exactly, because there is only a pole, and the cut on the negative real axis is no longer present.

\section{Zeros of fractional oscillations}

The fractional oscillations occupy a special place in the oscillatory theory and what is why. On the one hand, they have a decay like a relaxation. On the other hand they show themselves as oscillations. Asymptotically the fractional oscillations become vanishingly small with $t \rightarrow \infty$. However, the feature has a deep cornerstone. Their representation in the form of two contributions is especially important. The decomposition manifests a competition between two different dependencies. The harmonic oscillation exponentially decays on the background of a slow algebraic relaxation. Although at the beginning the oscillations prevail, the algebraic decay will survive them. As a result, the 
fractional oscillations have a finite number of damped oscillations as well as zeros.

Consider the problem in greater detail. Probably, Wiman was the first who has described the position of the Mittag-Leffler function zeros in the complex plane [15]. A finite number of the zeros has been established. Later the analysis was specified in [1]. The function $e_{\alpha}(t)$ demonstrates an odd number of zeros. The smallest zero lies in the interval $0<t<\pi /[\sin (\pi / \alpha)]$. The number of zeros strictly depends on the index $\alpha$. Increasing $\alpha$ more and more, we get more the number of zeros in the function $e_{\alpha}(t)$. For $\alpha=1$ the only zero is located in infinity. When $\alpha$ tends from 1 to 2 , the number of zeros increases so that for $\alpha=2$ their number becomes equal to infinity.

We provide a similar analysis to the function $i_{\alpha}(t)$. It is not evident that the function has a finite number of zeros for $1<\alpha<2$. At once it should be noticed that this function always is zero for $t=0$ under any value of $1 \leq \alpha \leq 2$. When $t$ is enough large, the zeros of $i_{\alpha}(t)$ are expected to be found approximately from the equation

$$
\frac{2}{\alpha} e^{t \cos (\pi / \alpha)} \approx \frac{t^{-\alpha / 2}}{\Gamma(1-\alpha / 2)},
$$

neglecting the oscillation factor in the contribution $q_{\alpha}(t)$. Putting now $\alpha=$ $1+\varepsilon$, the first-order approximation gives

$$
\cos (\pi / \alpha)=\cos [\pi /(1+\varepsilon)] \sim \cos [\pi(1-\varepsilon)] \sim-1
$$

Thus, the asymptotic position $T$ of the largest zero is defined by the relation

$$
e^{-T} \sim \frac{(1+\varepsilon)}{2 \Gamma(1 / 2+\varepsilon / 2)} T^{-1 / 2-\varepsilon / 2},
$$

which shows that $T$ tends to infinity as $\varepsilon \rightarrow 0$.

The function $i_{2}(t)$ has infinitely many zeros. Now let the index $\alpha$ be $2-\delta$. In the limit of the first-order approximation we can write

$$
\cos (\pi / \alpha)=\cos [\pi /(2-\delta)] \sim \cos [(\pi / 2)(1+\delta / 2)]=\sin (\pi \delta / 4) \sim \pi \delta / 4
$$

and

$$
\Gamma(1-\alpha / 2)=\Gamma(\delta / 2) \sim 2 / \delta
$$


In this case the asymptotic estimation for the largest zero $T$ reduces to the equation

$$
e^{-\pi \delta T / 4} \sim \frac{\delta(2-\delta)}{4} T^{-1+\delta / 2}
$$

from which one gets

$$
\pi \delta T / 4 \sim \ln (2 T / \delta)
$$

Since $\delta \rightarrow 0$, the value $T$ tends to infinity faster than $1 / \delta$. Moreover, the terms $\pi \delta T / 4$ and $\ln (2 T / \delta)$ are of the same order. Taking either $T \sim(a / \delta) \ln (2 / \delta)$ or $\delta \sim b \ln (T) / T$, where $a$ and $b$ are positive constants to be determined, we arrive at $a=b=8 / \pi$. The equivalent asymptotic expressions are

$$
\begin{aligned}
T & \sim \frac{8}{\pi \delta} \ln \left(\frac{2}{\delta}\right) \\
\delta & \sim \frac{8}{\pi} \frac{\ln T}{T} .
\end{aligned}
$$

In the limit $\alpha \rightarrow 2$ the period of $q_{\alpha}(t)$ becomes $2 \pi$. Consequently the number of zeros of $i_{\alpha}(t)$ tends to $N \sim T / \pi \rightarrow \infty$ as $\delta \rightarrow 0$.

\section{Conclusions}

We have considered the fractional oscillations allied with ordinary harmonic ones. The main feature of the fractional oscillations is that they have a finite number of zeros. This follows from the competition of two terms. One of them has an asymptotic behavior with an algebraic decay, and the other term contains a conventional harmonic oscillation vanishing in time because of an exponential decay. The second term decreases faster than this happens for the term with an algebraic decay. This important peculiarity of fractional oscillations is just reflected on the title of the paper. The analysis supports a key role of Mittag-Leffler functions. The fractional oscillations satisfy a linear fractional differential equation. We have derived it, using the method of the Laplace transform. Our analytical solutions are confirmed by a numerical treatment. 


\section{Acknowledgements}

The author wants to thanks Prof. Narahari Achar and Prof. George Zaslavsky for useful discussions on the subject.

\section{References}

[1] R. Gorenflo, F. Mainardi, Fractional oscillations and Mittag-Leffler functions, Proceedings of RAAM '96, Kuwait University (1996) 193.

[2] F. Mainardi, Chaos, Soliton \& Fractals 7 (1996) 1461.

[3] B. N. Narahari Achar, J. W. Hanneken, T. Enck, T. Clarke, Physica A297 (2001) 361.

[4] I. M. Sokolov, Phys.Rev. E63 (2001) 056111.

[5] A. A. Stanislavsky, Phys.Rev. E70 (2004) 051103.

[6] I. S. Gradshtein, I. M. Ryzhik, Tables of Integrals, Series, and Products, Acad. Press, New York, 1980.

[7] M. Abramowitz, I. A. Stegun, Handbook of Mathematical Functions, Dover, New York, 1972.

[8] K. S. Miller, S. G. Samko, Integr. Transf. and Spec. Func. 12(4) (2001) 389.

[9] M. M. Meerschaert, H.-P. Scheffler, J. Appl. Probab. 41 (2004) 623.

[10] A. A. Stanislavsky, Theor. and Math. Phys. 138 (2004) 418.

[11] Yu. Rabotnov, Creep problems in structural members, North-Holland, Amsterdam, 1969, p. 129. Originally published in Russian as: Polzuchest' Elementov Konstruktsii, Nauka, Moscow, 1966.

[12] G. Doetsch, Introduction to the Theory and Application of the Laplace Transformations, Springer-Verlag, Berlin, 1974.

[13] I. Podlubny, Solutions of linear fractional differential equations, In P. Rusev, I. Dimovski and V. Kiryakova (eds.), Transform Methods and Special Functions, Sofia 1994, Science Culture Technology, Singapore, 1995, pp. 227237.

[14] K. S. Miller, B. Ross, An Introduction to the Fractional Calculus and Fractional Differential Equations, Wiley, New York, 1993.

[15] A. Wiman, Acta Math. 29 (1905) 217. 